\title{
La typologie générale des constructions impersonnelles et les constructions impersonnelles du français
}

\author{
Denis Creissels \\ Université Lumière (Lyon 2) \\ denis.creissels@univ-lyon2.fr \\ http://deniscreissels.fr
}

\section{Introduction}

L'impersonnalité est un sujet qui a été largement débattu dans les travaux sur les langues d'Europe, notamment en linguistique française (mais aussi par exemple en linguistique russe, où il a donné lieu à une littérature particulièrement abondante - cf. Guiraud-Weber 1984). Toutefois, c'est seulement à date récente que l'impersonnalité a fait l'objet de recherches approfondies dans une perspective translinguistique ou typologique sortant du cadre des langues pour lesquelles l'impersonnalité constituait un thème de recherche traditionnel - cf. notamment Creissels (2007), Siewierska (2008), Malchukov \& Siewierska (2011). Ceci s'explique par une difficulté évidente à cerner une notion d'impersonnalité qui soit à la fois cohérente avec la façon dont cette notion a déjà été utilisée dans un certain nombre de langues et applicable à de nouvelles langues présentant des types différents d'organisation des constructions prédicatives.

Dans la perspective de dégager une notion translinguistique de l'impersonnalité, on doit tout d'abord observer que parmi les définitions couramment proposées dans les manuels de grammaire, certaines sont manifestement trop vagues et trop déconnectées des phénomènes qu'elles ont la prétention de délimiter pour être d'une quelconque utilité. Par exemple, il est clair qui si on appliquait à la lettre une définition telle que 'Les verbes impersonnels servent à exprimer une action qui n'est pas réalisée par une personne', on aboutirait à une délimitation de l'impersonnel n'ayant pas grand-chose à voir avec ce que l'on entend généralement par là en grammaire française. D'autres définitions sont tout simplement inapplicables car logiquement incohérentes, comme les définitions qui posent d'abord qu'un verbe impersonnel s'emploie uniquement à la troisième personne pour préciser ensuite qu'un verbe peut être impersonnel de manière occasionnelle. Avec ce genre de pseudo-définitions, reconnaître une construction comme impersonnelle ne peut se faire que par référence à une tradition qui énumère de façon plus ou moins arbitraire les constructions rangées sous cette étiquette, sans expliquer vraiment ce qui peut justifier leur regroupement.

D'autres définitions sont parfaitement précises et opératoires, mais trop liées à des particularités d'une langue ou d'un ensemble limité de langues pour se prêter facilement à une reformulation permettant de les utiliser dans une perspective typologique. C'est notamment le cas de la définition de l'impersonnel français comme construction prédicative incluant un il explétif (ou non référentiel) : à condition de s'entendre sur les propriétés qui caractérisent le il explétif, cette définition est parfaitement opératoire pour le français, par contre elle ne dit rien de la possibilité de retrouver des phénomènes morphosyntaxiques comparables dans les 
langues qui n'ont pas un système d'indices de sujet clitiques distribués de la même façon qu'en français. C'est aussi le cas de la définition de l'impersonnel latin ou russe comme construction prédicative n'incluant aucun position disponible pour un terme au nominatif, qui est parfaitement adaptée au système de ces langues mais ne se prête pas de manière évidente à une généralisation à des langues qui n'ont pas un système casuel semblable jusque dans les détails à celui du latin ou du russe.

Un point crucial est que les définitions courantes de l'impersonnel, lorsqu'elles ne mentionnent pas directement des éléments morphologiques propres à une langue particulière, se réfèrent à la notion de sujet, dont le statut dans une perspective translinguistique est très problématique. La position défendue par des typologues comme Gilbert Lazard, Wiliam Croft ou Martin Haspelmath, ${ }^{1}$ position à laquelle je me rallie moi-même, est qu'il est vain de chercher à définir une notion universelle de sujet dont le sujet de chacune des langues pour lesquelles on utiliser traditionnellement cette notion serait une instanciation particulière. Autrement dit, c'est sur une autre base qu'il faut chercher à développer une typologie de la façon dont les langues organisent l'expression de la relation entre le verbe et les arguments avec lesquels il forme une construction prédicative, et notamment à s'interroger sur la façon dont les constructions désignées comme impersonnelles dans telle ou telle tradition grammaticale peuvent être prises en compte par une telle typologie.

\section{Examen critique de la notion de sujet dans une perspective typologique}

Dans les descriptions du français, à condition de mettre à part les pseudo-définitions qui ne font qu'entretenir la confusion entre les notions de sujet syntaxique, agent et thème/topique, on peut dire que le sujet est défini comme un terme nominal de la prédication verbale qui se distingue des autres par le fait qu'il peut être représenté par un indice clitique de la série je / tu / il et gouverne l'accord du verbe en personne. L'importance donnée au sujet délimité selon ce critère est justifiée par le fait qu'il présente un comportement particulier, qui contraste avec celui des termes nominaux qui n'ont pas cette propriété, dans toute une série de mécanismes syntaxiques. A quelques détails près, on retrouve dans les autres langues d'Europe (à l'exception notable du basque) cette association entre un terme de la prédication verbale encodé de manière particulière (que ce soit en termes d'accord du verbe ou en termes de marquage casuel, ou les deux) et un type particulier de comportement dans un ensemble comparable de mécanismes syntaxiques.

Le problème est que l'accord obligatoire du verbe avec un terme particulier de sa construction ne se rencontre que dans une partie des langues. Le critère d'un marquage casuel particulier caractérisant le sujet (le cas 'nominatif') est lui aussi de manière évidente impossible à généraliser. Quant aux propriétés syntaxiques couramment associées à l'accord du verbe ou au cas nominatif dans les langues d'Europe, leur relation avec les caractéristiques de codage des termes nominaux de la phrase est souvent complexe, et pour chacun des mécanismes syntaxiques invoqués dans telle ou telle langue pour justifier le statut à part accordé au sujet, on peut toujours trouver des langues dans lesquelles le mécanisme en question, ou bien n'existe pas, ou bien ne marque aucun contraste entre un terme de la construction prédicative qui aurait un statut privilégié et les autres.

Ceci dit, il est certain qu'en ce qui concerne les propriétés traditionnellement attachées à la notion de sujet dans les traditions grammaticales européennes, certaines configurations sont

\footnotetext{
${ }^{1}$ Cf. notamment Lazard (1994 : 100-125, 2003, 2006 : 172-181, 2008, 2009).
} 
incontestablement plus communes que d'autres dans les langues du monde, et beaucoup de configurations logiquement possibles ne sont pas attestées du tout. Mais le type de configuration auquel s'attache l'étiquette de sujet dans les traditions grammaticales européennes, tout en étant particulièrement banal dans les langues du monde, est loin d'être universel. De nombreuses langues présentent d'autres types de configurations dans lesquelles beaucoup de propriétés qui concourent à faire du sujet une notion cruciale dans la syntaxe de la quasi-totalité des langues d'Europe, ou bien sont totalement absentes, ou bien se distribuent d'une façon qui ne permet pas de reconnaître dans le système prédicatif de ces langues un rôle syntaxique vraiment comparable au sujet des langues d'Europe.

\section{Une approche typologique de la notion de construction impersonnelle}

Une fois posé que les fonctions grammaticales traditionnellement reconnues dans la description des langues ne fournissent pas une base adéquate pour une typologie de l'expression des relations entre le verbe et ses arguments, il convient donc de chercher un concept comparatif (au sens de Haspelmath 2010) qui puisse servir de base à une telle comparaison, et notamment fournir une définition de la notion de construction impersonnelle qui soit opératoire dans une perspective translinguistique.

\subsection{Les notions de base de la typologie des systèmes de codage argumental}

La notion généralement retenue par les typologues comme base d'une typologie de l'expression des relations entre le verbe et les termes nominaux de sa construction est la notion de 'construction transitive de base' (ou plus simplement 'construction transitive'). Une fois la notion de construction transitive définie en des termes qui assurent la possibilité d'identifier universellement une construction répondant à cette définition, les systèmes de codage argumental peuvent être caractérisés en termes d'alignement entre la construction transitive de base et les autres construction prédicatives possibles. ${ }^{2}$

La notion de construction transitive de base s'appuie sur la longue lignée des travaux qui, à la suite de Hopper \& Thomson (1980), ont montré l'importance de la notion de transitivité prototypique pour l'analyse des régularités translinguistiques dans les systèmes de codage des arguments. C'est notamment sur cette notion sémantique de transitivité prototypique qu'on peut définir sans circularité une notion de transitivité syntaxique valable pour toutes les langues, en dépit des variations évidentes dans les détails de ses manifestations.

Un point crucial est que, pour être opératoire, la notion de transitivité prototypique doit s'appuyer sur une définition étroite d'agent et patient, comme l'ont notamment commenté Givón (1984 \& 1990), Kittilä (2002) ou Næss (2007) : l'agent typique exerce de manière consciente, contrôlée et volontaire une activité orientée vers un autre participant, et le patient typique subit un changement d'état ou de position du fait de l'activité d'un agent.

Dans les définitions qui vont être données, ${ }^{3}$ il convient de rester attentif au fait que, dans la terminologie suivie ici, transitif et intransitif en tant que notions morphosyntaxiques ne se réfèrent pas au nombre d'arguments d'un verbe, mais à un type particulier de codage des arguments. Les verbes encodant des événements à un, deux ou trois participants essentiels

\footnotetext{
${ }^{2}$ On notera que chez Gilbert Lazard, qui est l'un de ceux qui ont le plus contribué au développement de cette approche, la construction transitive de base est désignée comme 'construction biactancielle majeure'.

${ }^{3}$ Pour une discussion détaillée de ces définitions, on pourra se reporter à Creissels (2016).
} 
seront plutôt désignés respectivement comme monovalents, bivalents et trivalents, un verbe sémantiquement bivalent n'étant pas nécessairement syntaxiquement transitif.

La délimitation de l'ensemble des verbes transitifs est spécifique de chaque langue et repose sur des critères formels, mais l'ensemble des verbes transitifs de chaque langue peut se définir comme incluant une classe sémantique particulière de verbes, les verbes transitifs prototypiques : un verbe transitif prototypique est un verbe sémantiquement bivalent dont la structure argumentale est compatible avec un degré maximum de transitivité sémantique

Ceci étant posé, on peut définir la construction transitive de base, ou en abrégé construction transitive, comme la construction sélectionnée par les verbes transitifs prototypiques. En français, il va de soi que cette définition s'applique à la construction dans laquelle un verbe se combine avec deux groupes nominaux dont la mise en forme correspond aux notions traditionnelles de sujet et objet direct, mais ne permet par contre pas de récupérer la notion traditionnelle de 'transitivité indirecte'.

Enfin, on peut définir comme suit la notion de verbe transitif : un verbe transitif est un verbe qui entre dans la même construction que les verbes transitifs prototypiques, c'est-à-dire un verbe dont le cadre de codage inclut deux termes encodés comme l'agent et le patient des verbes transitifs prototypiques.

Selon cette définition, l'étiquetage d'un verbe comme transitif est indépendant du nombre de participants essentiels dans l'événement qu'il dénote et de leurs rôles sémantiques, et dépend uniquement du fait que sa construction comporte ou non deux termes encodés de la même façon que les deux arguments des verbes transitifs prototypiques.

Par exemple, le verbe français voir n'est pas un verbe transitif prototypique (puisqu'il n'a pas pour arguments un agent et un patient, mais plutôt un expérient et un stimulus), mais son cadre de codage l'identifie comme transitif : l'expérient de voir est codé comme l'agent des verbes transitifs prototypiques (sujet), et le stimulus de voir est codé comme le patient des verbes transitifs prototypiques (objet direct). De même en basque, ikusi 'voir' un verbe transitif, car son cadre de codage, avec notamment l'expérient au cas ergatif, est le même que celui d'un verbe transitif prototypique comme puskatu 'casser' - ex. (1). Par contre, le verbe akhvakh harigurusa 'voir' n'est pas transitif, car son cadre de codage, avec l'expérient au cas datif, diffère du cadre de codage sélectionné en Akhvakh par les verbes transitifs prototypiques tels que biq'ōruLa 'casser', dont l'agent est au cas ergatif - ex. (2).

(1) Basque

(1a) Haurr-ek ispilu-a puskatu dute.

enfant-PL.ERG miroir-SG casser.ACP avoir.PRS.A:3PL.P:3SG

'Les enfants ont cassé le miroir.'

(1b) Haurr-ek ispilu-a ikusi dute.

enfant-PL.ERG miroir-SG voir.ACP avoir.PRS.A:3PL.P:3SG

'Les enfants ont vu le miroir.'

\footnotetext{
${ }^{4}$ Dans la littérature typologique, c'est généralement le terme de 'construction intransitive étendue' qui est utilisé pour les constructions souvent désignées en linguistique française comme 'transitives indirectes', dans lesquelles l'un des deux arguments d'un verbe sémantiquement bivalent reçoit un codage différent de celui qui caractérise le patient des verbes transitifs prototypiques.
} 
(2) Akhvakh (caucasique de l'est)

(2a) Mik'i-de istaka biq'wāri.

enfant-ERG verre casser.ACP

'L'enfant a cassé le verre.'

(2b) Mik'i-La istaka harigwari.

enfant-DAT verre voir.ACP

'L'enfant a vu le verre.'

Pour éviter toute ambigüité entre l'agent ou le patient prototypique et les termes d'une construction encodés de la même façon qu'un agent ou un patient, on peut convenir de désigner comme $\mathrm{A}$ et $\mathrm{P}$ les termes nominaux de constructions prédicatives encodés dans une langue donnée de la même façon que l'agent et le patient des verbes transitifs prototypiques, quel que puisse être leur rôle sémantique.

\subsection{La 'typologie de l'alignement' et la notion de construction impersonnelle}

Le terme de 'typologie de l'alignement' est celui couramment utilisé pour se référer à la branche de la typologie linguistique qui s'intéresse aux relations possibles, d'une langue à l'autre, entre la construction transitive au sens défini ci-dessus et les autres constructions prédicatives possibles. La notion de base de cette typologie est la caractérisation des verbes sémantiquement monovalents comme pouvant présenter un alignement de type nominatifaccusatif ou de type absolutif-ergatif selon que les caractéristiques de codage de leur unique argument (selon les langues: marquage casuel, accord du verbe, ordre linéaire des constituants) coïncident avec celles de celles de l'agent des verbes transitifs prototypiques (comme à l'exemple (3)) ou avec celles du patient (comme à l'exemple (4)).

(3) Russe

(3a) Devuška prišl-a.

fille(F) venir.PAS-SG.F

'La fille est venue.'

(3b) Doktor prišël-Ø.

docteur(M) venir.PAS-SG.M

'Le docteur est venu.'

(3c) Doktor vylečil-Ø devušku.

docteur(M) soigner.PAS-SG.M fille(F).ACC

'Le docteur a soigné la fille.' 
(4) Avar (caucasique de l'est)

(4a) Jas j-ač'ana.

fille(F) SG.F-venir.ACP

'La fille est venue.'

(4b) Was w-ač'ana.

garçon(M) SG.M-venir.ACP

'Le garçon est venu.'

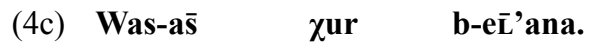

garçon(M)-ERG champ(N) SG.N-labourer.ACP

'Le garçon a labouré le champ.'

Une façon simple de généraliser à un maximum de langues la notion de construction impersonnelle consiste à s'appuyer sur une version de la typologie de l'alignement selon laquelle les langues sont caractérisées comme respectant plus ou moins ce que j'ai proposé d'appeler le principe du codage obligatoire (cf. Creissels 2018b). ${ }^{5}$ Dans une langue qui respecte strictement ce principe, il existe un type particulier de codage argumental qui doit être présent dans toute construction prédicative, avec deux possibilités logiques :

- dans une langue à codage $\mathrm{A}$ obligatoire, toute construction prédicative doit inclure un terme A, c'est-à-dire un terme codé de la même façon que l'agent des verbes transitifs prototypiques ;

- dans une langue à codage $\mathrm{P}$ obligatoire, toute construction prédicative doit inclure un terme $\mathrm{P}$, c'est-à-dire un terme codé de la même façon que l'agent des verbes transitifs prototypiques.

On peut observer alors que beaucoup de langues à travers le monde présentent une situation très proche de l'une des deux configurations idéales qui viennent d'être définies, sans toutefois que la coïncidence soit parfaite. En particulier, dans beaucoup de langues (et notamment dans la totalité des langues d'Europe à la seule exception du basque), les constructions prédicatives qui incluent un terme A sont très clairement prédominantes, mais il existe aussi de manière plus ou moins marginale des constructions prédicatives ne comportant aucun terme identifiable comme A. Cette situation est précisément celle des langues européennes dont les descriptions utilisent traditionnellement la notion de construction impersonnelle. On peut donc proposer la généralisation suivante de la notion de construction impersonnelle :

Dans une langue où la règle générale est que toute construction prédicative doit comporter un terme aux caractéristiques de codage identiques à celles de l'agent des verbes transitifs prototypiques, une construction impersonnelle est une construction prédicative qui s'écarte de la règle générale en ne comportant aucune place pour un terme aligné avec l'agent des verbes transitifs prototypiques.

\footnotetext{
${ }^{5}$ Cette notion est développée dans la linguistique générative sous le nom de paramètre du cas obligatoire (en anglais obligatory case parameter).
} 
Selon cette définition, la notion de construction impersonnelle n'est pas une notion universelle, mais une notion dont l'applicabilité à une langue est conditionnée par certains aspects de son système de codage argumental. Il n'est toutefois pas difficile de voir que, pour les langues auxquelles s'st intéressée la grammaire traditionnelle européenne, cette définition est équivalente à celles qui définissent une construction impersonnelle comme une construction ne comportant pas de terme sujet, ou comme comportant seulement un sujet 'explétif' (si du moins on s'en tient strictement à la définition du sujet en termes d'accord du verbe et/ou marquage casuel). Mais dans une perspective translinguistique, elle a l'avantage considérable de ne pas subordonner l'application de la notion de construction impersonnelle à la validité d'une notion dont le statut translinguistique est particulièrement problématique, comme c'est le cas pour la notion de sujet.

\section{Constructions impersonnelles et autres phénomènes couramment considérés comme relevant de l'impersonnalité}

A la section précédente, j'ai présente une possibilité de définir un concept comparatif de construction impersonnelle qui reprend un aspect important des définitions traditionnelles de l'impersonnel. Selon cette définition, dans une langue où la règle générale est que les constructions prédicatives incluent un terme codé de la même façon que l'agent des verbes transitifs prototypiques, une construction impersonnelle est une construction qui s'écarte de la norme en ne comportant pas de place pour un terme nominal codé de la même façon que l'agent d'un verbe transitif prototypique

La question qui doit maintenant être examinée est que cette définition, qui s'appuie sur une conception strictement morphosyntaxique de la notion de construction impersonnelle, laisse de côté un certain nombre de phénomènes souvent considérés par les grammairiens (et notamment par les grammairiens français) comme relevant de l'impersonnalité.

Dans le cas précis du français, la définition proposée à la section 3 ci-dessus s'applique essentiellement sinon exclusivement aux constructions dans lesquelles la position de l'indice de sujet immédiatement à gauche du verbe est occupée par le il que je qualifierai d'explétif (comme dans Il est venu plusieurs personnes ou Il est surprenant que Jean ne soit pas encore arrivé), caractérisé par les propriétés suivantes :

- le il explétif ne peut pas s'analyser comme représentant un constituant qui figure ou pourrait figurer en position détachée ;

- il est impossible de substituer au il explétif un autre indice de sujet ou un groupe nominal sans changer aussi le reste de la construction.

En effet, ce comportement particulier du il explétif permet de l'analyser comme ayant pour seule fonction de saturer la position structurelle de l'indice de sujet, dans une construction qui ne comporte pas de place pour un terme qui interagirait avec cette position de la façon qui caractérise le sujet/agent de la construction transitive.

Par contre, on ne peut pas faire la même analyse pour les phénomènes suivants, bien que la notion d'impersonnalité soit fréquemment évoquée à leur propos : 
- l'utilisation de l'indice de sujet de troisième personne masculin pluriel en valeur indéterminée, comme en (5a);

- l'utilisation d'indices de deuxième personne en valeur générique, comme en (5b);

- l'utilisation de l'indice de sujet on pour exprimer la référence vague ou générique à des humains, comme en $(5 \mathrm{c})$;

- l'utilisation de l'indice de sujet ça en valeur de référence vague, comme en (5d);

- l'utilisation de ça dans des constructions où ça semble commuter avec le il explétif, comme en (5e).

(5) Français

(5a) Ils ont annoncé du beau temps.

Ils ont encore augmenté les impôts.

(5b) Vous ne pouvez jamais savoir ce que la vie vous réserve.

(5c) On frappe à la porte.

On a toujours besoin d'un plus petit que soi.

\section{(5d) Ça crie chez les voisins.}

\section{(5e) C'est surprenant que Jean ne soit pas encore arrivé.}

Dans les cas illustrés en (5a-d), il est clair que tout se joue au niveau des propriétés référentielles de l'indice de sujet, et non pas au niveau de la construction. En effet, dans de tels cas, l'indice de sujet que l'on peut être tenté de qualifier d'impersonnel peut être replacé par un autre indice de sujet qui n'a rien d'impersonnel, ou par un groupe nominal sujet, sans qu'il n'y ait rien d'autre à modifier dans la construction.

Le cas de la construction illustrée en (5e) est un peu moins évident, du fait de la commutation apparente avec le il explétif. Mais en réalité, il y a toute une série de manipulations qui sont possibles pour cette construction mais pas pour la construction avec il, et qui conduisent à la conclusion qu'à la différence du il explétif, ça a le fonctionnement normal d'un indice de sujet représentant par cataphore un constituant détaché à droite :

- la construction avec ça est possible avec une intonation qui souligne le détachement (que l'on peut représenter à l'écrit par une virgule) - cf. (6a) ;

- dans la construction avec ça, le constituant représenté par ça peut aussi bien figurer en position détachée à gauche $-\mathrm{cf}$. (6b) ;

- dans la construction avec ça, le constituant représenté par ça peut rester implicite à condition que le contexte permette de le rétablir - cf. (6c).

(6) Français

(6a) C'est surprenant, que Jean ne soit pas encore arrivé.

(6b) Que Jean ne soit pas encore arrivé, c'est surprenant. 
(6c) - C'est surprenant que Jean ne soit pas encore arrivé. - Pourquoi dis-tu que c'est surprenant ?

Comme cela est indiqué en (7), aucune de ces manipulations n'est possible avec il, ce qui montre bien qu'en dépit de l'équivalence sémantique entre Il est surprenant que ... et C'est surprenant que ...., on a affaire à deux constructions différentes, l'une avec un indice de sujet qui a son fonctionnement normal, l'autre avec un indice de sujet détourné de son fonctionnement normal, qui ne fait rien de plus qu'occuper une position dont il marque le blocage.

(7) Français

(7a) *Il est surprenant, que Jean ne soit pas encore arrivé.

(7b) *Que Jean ne soit pas encore arrivé, il est surprenant.

(7c) - Il est surprenant que Jean ne soit pas encore arrivé. - *Pourquoi dis-tu qu'il est surprenant?

Une fois montrée la spécificité morphosyntaxique des constructions du français qui incluent le il explétif, qui sont les seules constructions de cette langue instanciant de manière indiscutable le concept comparatif de construction impersonnelle proposé à la section 3, on peut bien sûr se demander s'il y aurait une possibilité de réunir sous une même définition les constructions impersonnelles au sens de la définition proposée ici et les autres phénomènes couramment considérés par les grammairiens comme relevant de l'impersonnalité. Mais on voit mal comment aboutir à un tel résultat sans se baser sur des critères sémantiques plutôt que formels, et le problème est alors que si on veut rester cohérent, il faudra accepter de ranger à la rubrique de l'impersonnalité toutes sortes de phénomènes en plus de ceux couramment rangés à cette rubrique.

Par exemple, si on définit l'impersonnalité en termes de mise en arrière-plan de l'agent (ou du participant qui serait assimilé à l'agent dans une prédication verbale canonique), il n'y a aucune raison de ne pas considérer la totalité des constructions passives comme impersonnelles, ce qui va à l'encontre de la pratique traditionnelle consistant à distinguer le passif personnel du passif impersonnel : en tant que construction, le passif personnel, illustré en (8a), est une construction prédicative parfaitement canonique, mais où le rôle de sujet est assumé par un participant autre que celui qui assume la même fonction dans la construction active, tandis que dans le passif impersonnel illustré en (8b), il n'y a pas de sujet, et il a le comportement du il explétif des constructions impersonnelles.

(8) Français

(8a) Une décision a été prise.

(8b) Il a été pris une décision. 
Je ne chercherai pas à aller plus loin sur la question de savoir comment analyser les relations entre les constructions impersonnelles stricto sensu et d'autres phénomènes qui leur sont fonctionnellement apparentés. Pour le français, cette question est discutée en détail par Achard (2012) dans le cadre de la grammaire cognitive. Il est important d'observer que le titre même de son ouvrage, qui parle d'Impersonals and other defocusing constructions marque bien qu'il ne s'agit pas là de nier la spécificité des constructions impersonnelles, mais plutôt de les voir comme le «noyau dur» d'un domaine fonctionnel auquel participent d'autres constructions. Dans une perspective typologique, c'est une question qui a été abordée notamment par Siewierska (2008) et Malchukov et Ogawa (2011).

\section{Typologie des constructions impersonnelles}

Dans cette section, il s'agit d'esquisser une comparaison de la place qu'occupent, d'une langue à l'autre, les constructions qui peuvent être considérées comme des instanciations du concept comparatif défini à la section 3 .

Compte tenu des problèmes particuliers d'analyse qu'ils soulèvent, les impersonnels météorologiques sont laissés de côté dans cette section. La section 6 leur sera consacrée.

\subsection{Constructions impersonnelles lexicales}

On peut caractériser comme lexicales les constructions impersonnelles qui constituent la seule option possible pour un verbe donné avec une structure argumentale donnée (comme par exemple en français, falloir ou s'agir), ou bien qui varient avec une construction canonique sans aucune implication de type sémantique ou pragmatique (comme en français dans II semble que le feu ait pris / Le feu semble avoir pris).

A travers les langues, il est intéressant d'observer que les constructions impersonnelles lexicales s'observent typiquement avec des verbes présentant un certain type de signifié lexical.

\subsubsection{Impersonnels affectifs}

Dans pas mal de langues à travers le monde, et notamment dans beaucoup de langues européennes, les constructions impersonnelles lexicales s'observent typiquement parmi les verbes exprimant des états physiques ou psychiques affectant les humains. C'était le cas en latin (cf. Ernout \& Thomas 1951, Fedriani 2014) :

(9) Latin

$\begin{array}{lllll}\text {...ut } & \text { me } & \text { pigeat } & \text { stultitia } & \text { mea. } \\ \text { que } & \text { 1SG.ACC } & \text { regretter.SBJF.3SG } & \text { stupidité.SG.GEN } & \text { POSS:1SG.SG.F.GEN } \\ \text { ‘..pour que je regrette ma stupidité.' } & \end{array}$

C'est aussi le cas en russe, où par exemple (vy)rvat' s'utilise transitivement au sens d'arracher (10b), et impersonnellement au sens de vomir (10a), tandis que tošnit' 'avoir la nausée' existe exclusivement en construction impersonnelle (11a-b). 
(10) Russe

(10a) Rebënka rvët.

enfant.SG.ACC vomir.PRS.3SG

'L'enfant vomit.'

(10b) Rebënok rvët

stranicu.

enfant.SG arracher.PRS.3SG page.SG.ACC

'L'enfant arrache la page.'

(11) Russe

(11a) Menja tošnit.

1SG.ACC avoir_la_nausée.3SG

'J'ai la nausée.'
Menja
tošnit
ot ètogo.
1SG.ACC avoir_la_nausée.3SG
de DEM.SG.N.GEN
'J'en ai la nausée.'

Hors d'Europe, ce type de construction impersonnelle se rencontre par exemple en tamoul, ou encore en quechua, langues où la prédication canonique implique un terme $\mathrm{A}$ au cas nominatif et gouvernant l'accord du verbe de manière très semble à ce qui s'observe dans les langues indo-européennes anciennes :

(12) Tamoul (dravidien)

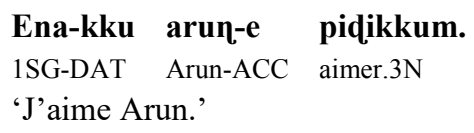

(13) Quechua d'Imbabura (Hermon 2001)

\author{
Ñuka-ta-ka chiri-wa-rka-mi. \\ 1SG-ACC-TOP avoir.froid-P:1-PST-VAL \\ 'J'ai eu froid.'
}

En français par contre, ce type d'emploi de la construction impersonnelle est très peu productif, et la tendance à l'éliminer est une constante dans l'évolution qui va du latin au français moderne : la construction impersonnelle de s'ennuyer de est pratiquement sortie de l'usage, et la construction impersonnelle de se souvenir de ne subsiste que dans un registre soutenu ou littéraire.

Dans une perspective de typologie diachronique, les constructions impersonnelles de ce type dans lesquelles l'expérient reçoit le même codage qu'un patient (comme en (9), (10), (11) et (13)), parfois désignées comme 'transimpersonnelles', ont comme origine probable des constructions transitives dont le terme A a disparu - cf. Creissels (2018b). 


\subsubsection{Verbes transitifs de possession utilisés impersonnellement comme verbes existentiels}

Les verbes d'existence, comme les verbes de localisation (dont ils diffèrent par une mise en perspective particulière de la relation prédicative), ont comme arguments un lieu pris comme repère (le 'fond') et une entité (la 'figure') située relativement à ce repère. Les verbes transitifs de possession (comme avoir en français) ont quant à eux comme arguments un possesseur et un possédé, auxquels ils assignent un codage identique à celui que les verbes transitifs prototypiques assignent respectivement à leur agent et à leur patient. Leur emploi impersonnel comme prédicats existentiels relève donc de la notion de construction impersonnelle lexicale, car il implique un changement de structure argumentale.

Contrairement à une idée reçue, les verbes transitifs de possession sont très courants dans les langues du monde, et l'emploi impersonnel de verbes transitifs de possession comme verbes d'existence n'est pas rare non plus, même s'il est très inégalement réparti selon les zones et les familles linguistiques. Par exemple, les langues de la famille atlantique (Afrique occidentale) ont pratiquement toutes des verbes transitifs de possession, et une bonne partie d'entre elles utilisent impersonnellement leur verbe de possession comme verbe d'existence cf. Creissels et al. (2015). C'est le cas notamment en wolof, où du fait du caractère facultatif de la présence d'un groupe nominal sujet, la phrase citée en (14) est ambigüe, avec une possibilité d'interprétation dans laquelle l'indice de 3ème personne réfère anaphoriquement à un possesseur sous-entendu (mais qui pourrait aussi être représenté par un groupe nominal précédant le verbe), et une deuxième possibilité d'interprétation dans laquelle il n'y a aucune possibilité d'introduire un groupe nominal sujet, et l'indice de troisième personne attaché au verbe se prête exactement à la même analyse que par exemple dans les constructions impersonnelles du latin ou du russe.

(14) Wolof (atlantique, Niger-Congo)

Am na ndox
avoir PRF.3SG eau
'Il/elle a de l'eau.' ou 'Il y a de l'eau.'

Cette ambigüité peut bien sûr être levée par la prise en considération du contexte, comme cela est illustré en (15) :

(15) Wolof (atlantique, Niger-Congo)

(15a) (Astu) am na jëkkër.

Astou avoir PRF.3SG mari

'Astou / elle a un mari.'

(15b) Am na ngelaw léegi.

avoir PRF.3SG vent aujourd'hui

'Il y a du vent aujourd'hui.' 
Ce phénomène est bien sûr attesté en français (il y a), mais avec en plus une particularité extrêmement rare typologiquement. En effet, pour naturel que cela puisse paraître, il n'est pas courant du tout que l'emploi impersonnel d'un verbe de possession comme verbe d'existence s'accompagne de la présence obligatoire d'un explétif locatif comme le y du français il y a. Je n'ai en tout cas trouvé aucune illustration de ce phénomène en dehors de la famille romane. Même parmi les langues d'Europe, aucun explétif locatif n'apparaît dans l'emploi existentiel du verbe 'avoir' en alémanique (es hot), en grec moderne ('́x $\chi \varepsilon \mathbf{\varepsilon})$ ou en bulgare (ima).

Pour plus de détails sur la typologie et la diachronie de l'emploi impersonnel de verbes transitifs de possession comme verbes existentiels, cf. Creissels (2018a)

\subsubsection{Construction impersonnelle propre à certains verbes modaux}

A l'instar du verbe français falloir construit avec un infinitif (16a) ou une complétive au subjonctif (16b), il n'est pas rare à travers les langues que des verbes de sens modal aient comme particularité lexicale d'appeler une construction impersonnelle, ou que des verbes qui ont un autre sens par ailleurs aient un emploi impersonnel dans une construction à valeur modale.

\section{(16) Français}

\section{(16a) Il me faut parler.}

\section{(16b) Il faut que je parle.}

Par exemple en tamoul (dravidien), mudiyum 'pouvoir' existe seulement à la forme de 3ème personne neutre. Ce verbe a nécessairement pour complément un infinitif, et le groupe nominal représentant la personne dont on prédique la capacité à faire quelque chose peut facultativement être au cas nominatif (mais sans gouverner l'accord du verbe), comme en (17a), ou bien au cas instrumental, comme en (17b).

(17) Tamoul (dravidien)

(17a) naan vara mudiyum.

1SG venir.INF pouvoir.3N

'Je peux venir.'

(17b) ennaale vara mudiyum.

1SG.INS venir.INF pouvoir.3N

'Je peux venir.'

On peut aussi citer le finnois, ou le verbe 'falloir' fait partie d'une classe limitée de verbes dont la construction comporte un groupe nominal au cas génitif et un infinitif. 
(18) Finnois (ouralien, Sands \& Campbell 2001)

$\begin{array}{lll}\text { Sinun pitää } & \text { mennä } \\ \text { 2SG.GEN } & \text { falloir.3SG } & \text { aller.INF } \\ \text { 'Tu dois partir.' } & \end{array}$

\subsubsection{Constructions impersonnelles liées à la présence d'un argument phrastique}

Ce type de construction impersonnelle est courant à travers les langues, et il est notamment largement attesté en français. Le cas du verbe apparaître fournit une illustration simple, puisque ce verbe peut avoir une construction canonique lorsque son argument prend la forme d'un groupe nominal (comme en (19a)), alors que seule la construction impersonnelle est possible s'il prend la forme d'une phrase (comme en (19b), cf. l'inacceptabilité de (19c)).

\section{(19) Français}

\section{(19a) Le soleil est apparu à travers les nuages.}

\section{(19b) Il est apparu que l'enfant mentait.}

\section{(19c) *Que l'enfant mentait est apparu.}

\subsubsection{Constructions impersonnelles conditionnées par l'animéité}

Beaucoup de langues ont des restrictions plus ou moins fortes sur la possibilité d'encoder des participants inanimés de la même façon que l'agent des verbes transitifs prototypiques. Dans le cas du russe, il existe une sous-classe de verbes transitifs, dont un représentant typique est razbit' (détruire), qui ont la possibilité d'une construction alternative lorsque le terme A ne représente pas un agent animé, mais une force inanimée. Il s'agit d'une construction impersonnelle dans laquelle le groupe nominal représentant la force qui déclenche l'événement est au cas instrumental, tandis que celui qui représente le patient est au même cas accusatif que dans une prédication transitive canonique.

(20) Russe

(20a) Molnija razbila stenu.

foudre(F) détruire.PAS.SG.F mur(F).ACC

'La foudre a détruit le mur.' (construction transitive canonique)

(20b) Stenu razbilo molniej.

mur(F).ACC détruire.PAS.SG.N foudre(F).INS

'La foudre a détruit le mur.' (construction impersonnelle) 


\subsection{Impersonnels modaux}

Nous avons vu en 5.1.3 qu'il n'est pas rare que des verbes de sens modal aient comme particularité lexicale d'appeler une construction impersonnelle. Il peut arriver aussi qu'un mécanisme régulier d'expression d'une valeur modale implique l'utilisation d'une forme verbale particulière allant de pair avec une construction impersonnelle.

Le russe a deux constructions de ce type. Dans celle illustrée en (21), le verbe est à l'infinitif, alors que dans celle illustrée en (22), le verbe est à la forme moyenne (forme marquée par le suffixe -sja, réflexe du pronom réfléchi indo-européen *se). Dans les deux cas, l'argument qui serait au cas nominatif dans la prédication verbale canonique est au cas datif.

(21) Russe

(21a) Čto my delaem?

quoi.ACC 1PL faire.PRS.1PL

'Que faisons-nous ?' (prédication canonique)

(21b) Čto nam delat'?

quoi.ACC 1PL.DAT faire.INF

'Que devons/pouvons-nous faire?' (impersonnel modal)

(22) Russe

(22a) Ja ne splju.

1 SG NEG dormir.PRS.1SG

'Je ne dors pas.' (prédication canonique)

(22b) Mne ne spit-sja.

1SG.DAT NEG dormir.PRS.3SG-REFL

'Je n'arrive pas à dormir' (impersonnel modal)

On peut citer aussi le cas du suffixe désidératif -naya- en quechua. En effet, en présence de ce suffixe, le verbe présente invariablement un indice de sujet de 3ème personne, qui doit être analysé comme explétif, tandis que l'argument qui serait le sujet dans une prédication canonique apparaît au cas accusatif - Hermon 2001. Ce suffixe ne change rien au reste de la construction du verbe, ce qui fait qu'avec les verbes transitifs, il provoque l'apparition d'une construction à deux accusatifs:

(23) Quechua d'Imbabura (Hermon 2001)

Ñuka-ta-ka aycha-ta miyu-naya-rka.

1SG-ACC-TOP viande-ACC manger-DESID-PAS.3

'Je veux manger de la viande.' 


\subsection{Constructions impersonnelles conditionnées pragmatiquement}

A cette rubrique comme à la précédente, il s'agit de constructions impersonnelles qui sont en concurrence avec une construction prédicative canonique pour toute une classe de verbes. La différence est que les constructions impersonnelles qui vont être examinées n'impliquent ni une modification de la structure argumentale, ni l'expression d'une valeur modale particulière. En effet, leur conditionnement met uniquement en jeu l'expression d'une valeur pragmatique qu'on peut résumer comme mise en retrait de l'argument qui serait sélectionné comme terme A dans une prédication canonique. Du point de vue formel, on peut distinguer selon que la construction impersonnelle met en jeu ou non une forme dérivée du verbe.

\subsubsection{Impersonnels pragmatiques morphologiquement non marqués}

Le phénomène évoqué à cette section concerne typiquement les verbes intransitifs dans des langues où l'ordre canonique des constituants dans la construction transitive est AVP (ou SVO dans la tradition directement issue des travaux de Greenberg sur l'ordre des mots). En effet, dans de telles langues, on observe souvent la possibilité d'une construction alternative discursivement marquée, souvent désignée comme 'inversion présentationnelle', ${ }^{6}$ dans laquelle le terme correspondant au A de la prédication canonique est en position post-verbale. Il est bien connu que, selon les langues, il peut y avoir des restrictions plus ou moins fortes sur la possibilité d'utiliser les verbes intransitifs dits 'inergatifs' dans cette construction, et inversement il peut y avoir sous certaines conditions la possibilité d'étendre la construction à inversion présentationnelle aux verbes transitifs - sur le français, cf. notamment Cummins (2000). Formellement parlant, il y a aussi d'importantes variations dans le fait que l'argument A déplacé en position post-verbale conserve ou non les caractéristiques du terme A de la prédication canonique.

Le français et le tswana (bantou) illustrent deux cas d'inversion présentationnelle dans lesquels l'argument A déplacé en position post-verbale perd toutes les propriétés qui caractérisent le terme A d'une prédication canonique (notamment la relation d'accord avec le verbe) et présente un comportement qui est plus proche de celui du terme $\mathrm{P}$ d'une prédication canonique (phénomène évoqué par le titre du célèbre article de Lambrecht 'When subjects behave like objects'). Les constructions à inversion présentationnelle du français et du tswana sont formellement tout à fait comparables, il est toutefois intéressant d'observer qu'en tswana, à la différence du français, tous les verbes intransitifs ont également accès à cette construction.

\section{(24) Français}

(24a) Deux femmes sont venues. (prédication canonique)

(24b) Il est venu deux femmes. (inversion présentationnelle)

\footnotetext{
${ }^{6}$ Les termes utilisés pour se référer à ce type de construction varient beaucoup d'un auteur à l'autre. On parle notamment souvent de 'sentence focus construction' - cf. Lambrecht (2000)
} 
(25) Tswana (bantou)

(25a) Bà-símàní 'bá-tłáà-bî:n-à.

CL2-garçon CL2-FUT-danser-FV

'Les garçons danseront.' (prédication canonique)

\section{(25b) Xó-tláà-bín-á bà-símà:ní.}

CL17-FUT-danser-FV CL2-garçon

litt. 'Il dansera des garçons.' (inversion présentationnelle)

Dans d'autres langues (et ceci s'observe notamment aussi bien en roman qu'en bantou), le terme en position post-verbale dans la construction à inversion présentationnelle conserve la relation d'accord avec le verbe, et la construction ne peut donc pas être qualifiée d'impersonnelle au sens strict donné ici à ce terme. On observe toutefois que même lorsque la relation d'accord est maintenue, l'inversion présentationnelle peut affecter d'autres propriétés $\mathrm{du}$ terme A. Par exemple, dans l'inversion présentationnelle de l'espagnol, la relation d'accord avec le verbe est maintenue, mais on n'a plus les contraintes sur la présence d'un déterminant qui caractérisent le terme A en position canonique :

\section{(26) Espagnol}

\section{(26a) Los chicos llegaron.}

D.M.PL garçon.PL. arriver.PRET.3PL

'Les garçons sont arrivés.'

\section{(26b) *Chicos llegaron.}

garçon.PL. arriver.PRET.3PL

\section{(26c) Llegaron chicos.}

arriver.PRET.3PL garçon.PL.

'Il est arrivé des garçons.'

Dans une langue comme le russe, la flexibilité de l'ordre des constituants est telle que cela n'aurait pas beaucoup de sens de chercher à reconnaitre une construction à inversion présentationnelle en tous points semblable à celles évoquées ci-dessus. Il y a toutefois quelque chose d'analogue avec la construction des verbes intransitifs dite à 'génitif de négation', analysée en particulier par Babby (2001). Formellement, il s'agit d'une construction impersonnelle dans laquelle l'argument qui serait le terme A d'une prédication canonique est au cas génitif. Sémantiquement, le contraste avec la prédication canonique est comparable à celui observé dans les constructions à inversion présentationnelle :

\section{(27) Russe}

\section{(27a) Otvet ne prišël.}

réponse.SG NEG arriver.PFF.PAS.SG.M

'La réponse n'est pas arrivée.' 

(27b) Otveta ne prišlo.
réponse.SG.GEN NEG arriver.PFF.PAS.SG.N
'Il n'est pas arrivé de réponse.'

\subsubsection{Emploi impersonnel de formes passives}

On peut définir de manière générale les formes verbales passives comme des formes verbales qui se prêtent à une construction dans laquelle le participant représenté par le terme A de la construction transitive est mis en retrait (qu'il soit absent ou bien présent sous forme de terme oblique) tandis que le participant représenté par le terme $\mathrm{P}$ de la construction passive prend les caractéristiques de codage de l'unique terme nucléaire d'une construction intransitive - ce qui, dans les langues à codage $\mathrm{A}$ obligatoire comme le français, signifie que son codage s'aligne sur celui du terme A d'une construction transitive.

Dans beaucoup de langues à travers le monde, les formes verbales qui répondent à cette définition de formes verbales passives ont aussi une possibilité de construction impersonnelle dans laquelle le terme A de la construction transitive est également mis en retrait, mais le terme $\mathrm{P}$ n'est en rien 'promu' et garde le même codage que dans la construction transitive.

Un tel emploi de formes verbales passives existe en français :

\section{(28) Français}

(28a) Le président a pris une décision. (construction transitive)

(28b) Une décision a été prise (par le président). (passif avec 'promotion' de l'argument $\mathrm{P}$ )

(28c) Il a été pris une décision. (passif impersonnel)

Il y a toutefois un contraste important entre le passif impersonnel du français et le passif impersonnel de nombreuses langues à travers le monde, dans le fait qu'en français, l'emploi impersonnel de formes passives ne s'étend pas (ou seulement de façon très marginale) aux verbes intransitifs. Par exemple en tswana, (29c) illustre une passif impersonnel de verbe transitif très semblable au passif impersonnel du français, tandis que (30b) montre l'utilisation de la même dérivation morphologique pour mettre en retrait l'unique argument nucléaire d'un verbe intransitif.

(29) Tswana (bantou, Niger-Congo)

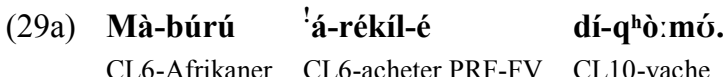

'Les Afrikaners ont acheté les/des vaches.' 
(29b) Dì-qhòmó dí-rèkíl-w-è (kí Mà-bû:rù).

CL.10-vache CL10-acheter.PRF-PSF-FV par CL6-Afrikaner

'Les vaches ont été achetées (par les Afrikaners)' (passif canonique)

(29c) Xó-rékíl-w-é dí- q⿳ò̀mó.

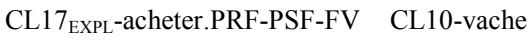

'Il a été acheté des vaches.' (passif impersonnel)

(30) Tswana (bantou, Niger-Congo)

(30a) Kítsó ó-bùî:l-è.

(CL1)Kitso CL1-parler.PRF-FV

'Kitso a parlé.'

(30b) Xó-bùî:l-w-è.

CL17 EXPL-parler.PRF-PSF-FV

'On a parlé.', lit. 'Il a été parlé.' (passif impersonnel)

Compte tenu de cette limitation à l'emploi impersonnel des formes passives du français, on peut considérer qu'en français, à la différence d'autres langues qui ont aussi un emploi impersonnel de formes passives, il ne s'agit pas à proprement parler d'une construction particulière, mais plutôt d'un phénomène qu'on peut décrire en posant que les formes passives se prêtent à l'inversion présentationnelle de la même façon que des formes intransitives non dérivées : Il a été pris une décision est à Une décision a été prise ce que Il est venu deux personnes est à Deux personnes sont venues.

\subsubsection{Emploi impersonnel de formes moyennes}

L'emploi impersonnel de formes moyennes est aussi un phénomène courant dans les langues du monde, qui s'observe notamment dans pas mal de langues européennes avec les formes moyennes issues de la grammaticalisation du réfléchi indo-européen *se.

Par exemple en espagnol, (31a) est une construction transitive, et (31b) sa variante détransitivisée par l'utilisation d'une forme moyenne, avec deux interprétations possibles (réciproque et passive). Quant à (31c), il s'agit d'un emploi impersonnel de la même forme moyenne, dans lequel le terme $\mathrm{P}$ de la construction transitive se retrouve avec exactement le même codage que dans la construction transitive, l'expression de l'argument A étant par contre bloquée.

(31) Espagnol

$\begin{array}{llllll}\text { (31a) El } & \text { policía } & \text { encontró } & \text { a } & \text { los } & \text { ladrones. } \\ & \text { D.SG.M policier } & \text { trouver.PRET.3SG } & \text { ACC } & \text { D.PL.M } & \text { voleur.PL } \\ \text { 'Le policier a trouvé les voleurs.' } & & & \end{array}$


(31b) Se encontraron los ladrones.

MOY trouver.PRET.3PL D.PL.M voleur.PL

'Les voleurs se sont rencontrés.' ou 'Les voleurs ont été trouvés.'

(31c) Se encontró a los ladrones.

MOY trouver.PRET.3SG ACC D.PL.M voleur.PL

'On a trouvé les voleurs.', litt. 'Il s'est trouvé les voleurs.'

Le même phénomène s'observe en italien :

Italien

Si accusò i colpevoli.

MOY accuser.PRET.3SG D.PL.M coupable.PL

'On a accusé les coupables.' lit. 'Il s'est accusé les coupables.'
(32b) Si legge $\quad$ i giornali prima di cominciare
MOY trouver.PRET.3SG D.PL.M journal.PL avant de commencer.INF
a lavorare
à travailler.INF
'On lit les journaux avant de commencer à travailler.'
lit. 'Il se lit les journaux avant de commencer à travailler.'

En français, on peut aussi trouver des emplois impersonnels de formes moyennes (cf. par exemple Il s'est vendu beaucoup de livres), mais on peut faire à leur sujet la même remarque que pour l'emploi impersonnel de formes passives : à la différence de l'espagnol ou de l'italien, plutôt que d'une construction spéciale, il s'agit simplement de l'aptitude des formes moyennes à se prêter à l'inversion présentationnelle de la même façon que des formes intransitives non dérivées (Il s'est vendu beaucoup de livres est à Beaucoup de livres se sont vendus ce que Il est venu deux personnes est à Deux personnes sont venues). Cette analyse n'est pas transposable à l'espagnol ou à l'italien pour la simple raison que dans ces langues, l'inversion présentationnelle maintient l'accord du verbe avec le terme en position post-verbale.

\subsubsection{Formes verbales dérivées spécialisées en emploi impersonnel}

Il existe dans certaines langues (mais pas en français) des formes verbales dérivées qui impliquent la mise en retrait de l'argument encodé comme terme A dans la construction de la forme dérivée du même verbe sans rien changer au reste de la construction. A propos de ces formes, il règne au niveau de la terminologie une certaine confusion. En effet, elles sont souvent désignées comme 'passives', alors même qu'elles ne se prêtent pas à des constructions passives canoniques avec 'promotion' du terme P. On devrait donc plutôt parler de voix impersonnelle. Selon les langues, avec de telles formes, l'argument encodé comme terme A d'une prédication canonique reste obligatoirement non exprimé, ou bien peut apparaître comme un oblique. De formes dérivées du verbe spécialisées en emploi impersonnel se rencontrent notamment en finnois, en polonais et dans les langues celtiques. 
(33) Finnois (ouralien)
Minä tunne-n
sinut.
1SG connaître.PRS-1SG 2SG.ACC
'Je te connais.'

$\begin{array}{lll}\text { Sinut } & \text { tunne-taan } & \text { siellä. } \\ \text { 2SG.ACC } & \text { connaître-IMPERS.PRS } & \text { là-bas } \\ \text { 'On te connaît là-bas.' } & \end{array}$

Sémantiquement, la forme impersonnelle du verbe en finnois est équivalente au français on, mais structurellement, on occupe une position d'indice de sujet, et la construction elle-même n'a rien qui la distingue d'une prédication canonique, tandis qu'en finnois, certaines formes verbales (notamment les formes négatives du verbe) excluent sans ambigüité l'analyse du morphème glosé IMPERS ci-dessus comme un indice de sujet. En finnois, on a affaire à la formation d'une base verbale dérivée qui a la propriété de bloquer l'expression d'un des arguments du verbe sans modifier le reste de sa construction.

\section{La question des 'impersonnels météorologiques'}

Les phrases météorologiques du français telles que Il pleut ou Il fait froid semblent à première vue répondre à la définition d'une construction impersonnelle. Toutefois, un certain nombre d'études ont attiré l'attention sur des comportements qui distinguent le il météorologique du français du il explétif dans les constructions examinées jusqu'ici, et des observations qui vont dans le même sens (bien que différentes dans le détail) ont été faites sur le it météorologique de l'anglais. On peut mentionner par exemple la possibilité d'avoir en français familier un ça météorologique (Ça pleut) qui, à la différence du ça de c'est surprenant que..., ne semble impliquer aucun changement de construction par rapport à il. On peut aussi évoquer la possibilité qu'a le il météorologique de contrôler un infinitif (Il a plu avant de neiger), propriété dont est dépourvu le il explétif.

Ruwet (1990) propose une discussion extrêmement détaillée et pertinente de la question des phrases météorologiques, dans laquelle il montre bien la complexité du problème et argumente de façon convaincante contre les analyses qui veulent accorder au il météorologique une sorte de statut argumental. Je me contenterai ici de reprendre brièvement une des idées qu'il a développées dans cet article, à savoir l'intérêt d'un éclairage typologique pour comprendre où réside véritablement le problème.

Il est particulièrement intéressant d'observer les constructions attestées à travers les langues pour les phrases météorologiques dans lesquelles l'événement météorologique luimême n'est pas lexifié comme nom (du genre 'La pluie tombe' ou 'Le vent se lève'). Dans les phrases météorologiques dans lesquelles le phénomène météorologique n'est pas mentionné sous forme de nom, on peut selon les langues avoir des constructions où ne figure aucun nom (et qu'on sera donc tenté de qualifier d'impersonnelles) mais aussi très souvent (notamment, mais pas seulement, dans les langues d'Afrique subsaharienne) des constructions incluant un nom traité en apparence de la même façon que l'argument d'un verbe monovalent. Toutefois, le nom en question est toujours quelque chose comme 'ciel', 'atmosphère', 'endroit', ou 
'Dieu', et crucialement, sa présence doit s'analyser comme le résultat d'une convention (variable d'une langue à l'autre) plutôt que d'un véritable choix, car à partir du moment où le verbe est employé en référence à un événement météorologique, la présence ou l'absence d'un tel nom n'a jamais d'incidence sur la signification. En d'autres termes, ce nom occupe superficiellement une position argumentale sans être véritablement un argument au sens logique du terme.

Par exemple, en balant ganja (atlantique), langue où l'indexation du sujet marque des distinctions de genre (ou de 'classe' dans la terminologie traditionnelle des africanistes), le verbe tob 'pleuvoir' peut ou bien se construire avec le nom Hàalá 'Dieu', ou bien former une phrase à lui seul en s'attachant l'indice de sujet correspondant (classe HA, c'est-à-dire 'humain singulier'). Superficiellement, c'est une prédication verbale qui semble parfaitement canonique. Toutefois, 'Dieu' et l'indice de sujet correspondant dans la construction apparemment canonique du balant ganja sont tout aussi figés que le il météorologique du français : par exemple, il est tout simplement inconcevable de focaliser le pseudo-sujet de cette construction (*c'est Dieu qui pleut) ou de lui substituer un pronom interrogatif (*qui estce qui pleur?).

Balant ganja (atlantique)

\section{(34a) Hàalá n-tớbì.}

Dieu INACP-pleuvoir

'Il va pleuvoir.' litt. 'Dieu va pleuvoir.'

\section{(34b) À-n-tóbì.}

CLha-INACP-pleuvoir

'Il va pleuvoir.'

De telles observations suggèrent que le il des expressions météorologiques du français est fondamentalement différent du il explétif, même s'il partage avec lui certaines propriétés (la plus évidente étant de ne pas pouvoir être analysé comme représentant un nom). En effet, le il explétif marque le choix d'une construction qui s'écarte de la norme par le fait que, parmi les arguments disponibles, aucun ne peut être codé de la même façon que l'agent des verbes transitifs prototypiques. Par contre, un verbe météorologique est fondamentalement avalent, au sens où, sémantiquement, aucune précision sur d'éventuels participants n'est requise pour passer du signifié lexical du verbe à un contenu propositionnel. Autrement dit, avec les verbes météorologiques, une construction prédicative canonique avec un argument codé de la même façon que l'agent des verbes transitifs prototypiques est tout simplement inconcevable. Mais les verbes météorologiques n'ont pas non plus une construction qui mériterait véritablement d'être qualifiée d'impersonnelle, si on estime essentiel dans la notion de construction impersonnelle la possibilité d'au moins imaginer une construction alternative avec un terme A représentant un argument.

Dit de manière un peu différente, le il météorologique du français, à la différence du il explétif, n'est pas motivé par le choix d'une construction n'incluant aucun sujet, mais par l'impossibilité d'utiliser la position sujet pour l'expression canonique d'un participant, du fait de l'absence de structure argumentale qui caractérise les verbes météorologiques. A la différence du il explétif, qui marque le blocage de la position sujet, on peut analyser le il 
météorologique comme un simple sujet par défaut, au même titre par exemple que le nom 'Dieu' dans l'exemple balant (34a).

A partir de là, on peut comprendre que chaque langue va régler à sa façon le choix d'un terme A par défaut pour les verbes météorologiques, ainsi que la codification de ses propriétés morphosyntaxiques. Selon les langues, la construction des verbes météorologiques pourra ainsi ressembler plus ou moins fortement à une construction impersonnelle proprement dite (si le sujet par défaut des verbes météorologiques est un pronom ou indice qui fonctionne par ailleurs comme explétif dans les véritables constructions impersonnelles), ou au contraire avoir l'apparence d'une construction prédicative canonique (si le sujet par défaut des verbes météorologiques est un nom). Dans le cas du français, cette analyse rend notamment compte de la possibilité de substituer ça au il météorologique sans rien changer au reste de la construction, alors que, comme cela a été rappelé ci-dessus, l'apparente commutation que l'on observe parfois entre le il explétif et ça implique en réalité un changement de construction.

\section{Conclusion}

Dans cet exposé, j'ai d'abord essayé de montrer comment on peut reprendre la notion traditionnelle de construction impersonnelle pour en tirer un concept comparatif applicable à toutes les langues proches du prototype de langue 'à codage A obligatoire', quels que puissent être par ailleurs les détails de la façon dont elles organisent l'expression des relations verbearguments.

J'ai ensuite argumenté qu'une définition rigoureuse de la notion de construction impersonnelle en termes morphosyntaxiques ne peut pas prétendre à englober tous les phénomènes que les grammairiens ont pu être tentés de ranger à la rubrique de l'impersonnalité, et qu'il convient plutôt de parler de parenté fonctionnelle entre des phénomènes morphosyntaxiques de nature différente.

A la section 5, j'ai esquissé une typologie générale des constructions impersonnelles au sens strict du terme, en montrant comment les constructions impersonnelles du français se situent par rapport à cette typologie.

Enfin, j'ai essayé de montrer comment l'approche typologique peut contribuer à une clarification de la question particulièrement délicate et controversée des 'impersonnels météorologiques', qui selon l'analyse que je propose ne relèvent pas véritablement de la notion de construction impersonnelle.

\section{Abréviations}

$\mathrm{A}=$ terme d'une construction prédicative encodé de la même façon que l'agent des verbes transitifs prototypiques, $\mathrm{ACC}=$ accusatif, $\mathrm{ACP}=$ accompli, $\mathrm{CL}=$ classe nominale, $\mathrm{D}=$ défini, $\mathrm{DAT}=$ datif, DEM = démonstratif, DESID = désidératif, ERG = ergatif, EXPL = explétif, $\mathrm{F}$ = féminin, FUT $=$ futur, FV $=$ finale verbale, GEN $=$ génitif, IMPERS $=$ impersonnel, INACP = inaccompli, INF = infinitif, INS = instrumental, $\mathrm{M}=$ masculin, $\mathrm{MOY}=$ moyen, $\mathrm{N}=$ neutre, $\mathrm{NEG}=$ négatif, $\mathrm{P}=$ terme d'une construction prédicative encodé de la même façon que le patient des verbes transitifs prototypiques, $\mathrm{PAS}=$ passé, $\mathrm{PFF}=$ perfectif, $\mathrm{PL}=$ pluriel, $\mathrm{POSS}$ $=$ possessif, $\mathrm{PRESENT}=$ présentatif, $\mathrm{PRET}=$ prétérit, $\mathrm{PRF}=$ parfait, $\mathrm{PRS}=$ présent, $\mathrm{PSF}=$ passif, $\mathrm{REFL}=$ réfléchi, $\mathrm{SBJF}=$ subjonctif, $\mathrm{SG}=$ singulier, $\mathrm{TOP}=$ topique, $\mathrm{VAL}=$ validateur. 


\section{Références}

Babby, L. 2001. The genitive of negation: a unified analysis. In Franks S., T. King \& M. Yadroff (eds.), Formal approaches to Slavic linguistics, The Bloomington meeting 2000. Ann Arbor: Michigan Slavic Publications. 39-55.

Creissels, Denis. 2007. Impersonal and anti-impersonal constructions: a typological approach. Ms, Université de Lyon. http://deniscreissels.fr.

Creissels, Denis. 2016. Transitivity, valency and voice. Cours à l'École d’Été Européenne de Typologie Linguistique (Porquerolles, septembre 2016). http://deniscreissels.fr.

Creissels, Denis. 2018a. Existential predication and transposssessive constructions in typological and diachronic perspective. Annual meeting of the Deutsche Gesellschaft für Sprachwissenschaft. Stuttgart, 7-9 March 2018.

http://www.deniscreissels.fr/public/Creissels-Exist.Transposs.2018.pdf

Creissels, Denis. 2018b. The Obligatory Coding Principle in diachronic perspective. In Sonia Cristofaro \& Fernando Zúñiga (eds.). Typological hierarchies in synchrony and diachrony. John Benjamins. 61-112.

http://www.deniscreissels.fr/public/Creissels-

Obligatory_Coding_Principle in diachronic perspective.pdf

Creissels, Denis, Sokhna Bao Diop, Alain-Christian Bassène, Mame Thierno Cissé, Alexander Cobbinah, El Hadji Dieye, Dame Ndao, Sylvie Nouguier-Voisin, Nicolas Quint, Marie Renaudier, Adjaratou Sall \& Guillaume Segerer. 2015. L'impersonnalité dans les langues de la région sénégambienne. Africana Linguistica 21. 29-86.

http://www.deniscreissels.fr/public/Creissels_et_al-Impers.Sen.pdf

Cummins, S. 2000. The Unaccusative Hypothesis and the Impersonal Construction of French. Canadian Journal of Linguistics / Revue canadienne de linguistique 45(3-4). 225-251.

Dixon, Robert M. W. \& Alexandra Y. Aikhenvald. 2000. Introduction. In Robert M. W. Dixon \& Alexandra Y. Aikhenvald (eds.), Changing Valency: Case Studies in Transitivity. Cambridge: Cambridge University Press. 1-29.

Ernout, A. \& F. Thomas. 1951. Syntaxe latine. Paris: Klincksieck.

Fedriani, Chiara. 2014. Experiential constructions in Latin: A synchronic and diachronic study. Bril.

Givón, Talmy. 1984. Syntax: a Functional-typological Introduction 1. Amsterdam: John Benjamins.

Givón, Talmy. 1990. Syntax: a Functional-typological Introduction 2. Amsterdam: John Benjamins.

Guiraud-Weber, Marguerite. 1984. Les propositions sans nominatif en russe moderne. Paris : Institut d'Etudes Slaves.

Haspelmath, Martin. 2010. Comparative concepts and descriptive categories in crosslinguistic studies. Language 86(3). 663-687.

Hériau, M. 1980. Le verbe impersonnel en français moderne. Lille: Atelier de reproduction de theses, Université de Lille III.

Hopper Paul J. \& Sandra A. Thompson. 1980. Transitivity in grammar and discourse. Language 56(2). 251-299.

Kittilä Seppo. 2002. Transitivity : towards a comprehensive typology. Turku: Åbo Akademis Tryckeri. 
Lambrecht, Knud. 2000. When subjects behave like objects: an analysis of the merging of $\mathrm{S}$ and $\mathrm{O}$ in sentence focus constructions across languages. Studies in Language 24. 611-682.

Lazard, Gilbert. 1994. L'actance. Paris : PUF.

Lazard, Gilbert. 1997. Review of Ergativity by R. M. W. Dixon. Linguistic Typology 1. 243268.

Lazard, Gilbert. 2003. Le sujet en perspective interlinguistique. in J.-M. Merle (éd.), Le sujet. Gap-Paris : Ophrys. 15-28.

Lazard, Gilbert. 2006. La quête des invariants interlangues. La linguistique est-elle une science?, Paris : Champion.

Lazard, Gilbert. 2008. Nouvelles remarques au sujet du sujet. BSL 103(1). 111-121.

Lazard, Gilbert. 2009. Qu'est-ce qu'un sujet ? La linguistique 45(1). 151-158.

Malchukov, Andrej \& Akio Ogawa. 2011. 'Towards a typology of impersonal constructions: A semanctic map approach'. In Andrej Malchukov \& Anna Siewierska (eds.), Impersonal constructions, a cross-linguistic perspective. 19-56. Amsterdam / Philadelphia: John Benjamins.

Malchukov, Andrej \& Anna Siewierska (eds.). 2011. Impersonal constructions, a crosslinguistic perspective. 19-56. Amsterdam / Philadelphia: John Benjamins.

Næss Åshild, 2007, Prototypical Transitivity, Amsterdam and Philadelphia, John Benjamins.

Ruwet, Nicolas. 1990. Des expressions météorologiques. Le Français Moderne 58. 43-97.

Siewierka, Anna. 2008. 'Introduction: Impersonalization: An agent-based vs. a subject-based perspective'. Transactions of the Philological Society 106(2). 115-137. (Special issue on Impersonal Constructions in Grammatical Theory, guest-edited by Anna Siewierska)

Tsunoda, Tasaku. 1985. Remarks on transitivity. Journal of Linguistics 21(2). 385-396. 